\title{
Local and regional patterns of fish assemblages in coastal lagoons surrounded by mangroves, Gulf of Tehuantepec in the south Pacific of Mexico
}

\author{
Emilio I. Romero-Berny ${ }^{1}$, Juan J. Schmitter-Soto ${ }^{2}$ \\ Ernesto Velázquez-Velázquez ${ }^{3}$ \& Adán E. Gómez-González ${ }^{3}$ \\ ${ }^{1}$ Centro de Investigaciones Costeras, Instituto de Ciencias Biológicas \\ Universidad de Ciencias y Artes de Chiapas, Tonalá, Chiapas, Mexico \\ ${ }^{2}$ Departamento de Sistemática y Ecología Acuática, El Colegio de la Frontera Sur \\ Unidad Chetumal, Chetumal, Quintana Roo, Mexico \\ ${ }^{3}$ Museo de Zoología, Instituto de Ciencias Biológicas, Universidad de Ciencias y Artes de Chiapas \\ Tuxtla Gutiérrez, Chiapas, Mexico \\ Corresponding author: Emilio I. Romero-Berny (emilio.romero@unicach.mx)
}

\begin{abstract}
We analyzed the local variation of fish assemblages in four coastal lagoon systems surrounded by mangroves, draining into the Gulf of Tehuantepec (Pacific versant of Mexico), and determined the spatial patterns of alpha, beta, and gamma diversity. Fish were sampled between 2004 and 2016 at 63 sites using cast nets. The collected data were supplemented with information obtained from published works for three other coastal lagoons for the regional analysis. Local richness was high ( 89 species in a total of 19,017 specimens in four systems). Locally, dissolved oxygen, depth, and distance to mangrove were variables that significantly affected richness and abundance of fish in one or more systems. The Chantuto-Panzacola system showed the highest richness, significantly different from the other systems, although the trophic groups were similar. Regionally, two, Istmo and Soconusco complexes were identified, whose turnover rate (0.36) and gamma diversity (176) increased from north to south. Fish species richness and abundance increased with growing mangrove area, both locally and regionally, making this a highly explanatory variable. The Gulf of Tehuantepec is an environmentally heterogeneous region, with ecological patterns defined according to the spatiotemporal scale, which should be considered in the delineation of ecoregions and coastal management planning.
\end{abstract}

Keywords: ichthyofauna; spatiotemporal variation; diversity; ecoregion; Tropical Eastern Pacific

\section{INTRODUCTION}

Much knowledge about biodiversity has been based on estimates of alpha diversity (local) and, to a lesser extent, beta diversity (level of species replacement among different environments), which are the components of gamma or total species diversity in a landscape (Magurran 1988). Many theories have been developed to explain the structure and maintenance of biotic communities within ecosystems. According to niche theory, physical-environmental factors and interactions between species are key to explaining communities' composition and taxonomic diversity (Keddy 1992, Chase \& Leibold 2003). On the other hand, the neutral theory considers that species can share a certain functional equivalence in a community whose structure will depend on stochastic, demographic, and biogeographic processes (Hubbell 2001, Bell et al. 2006). Far from a paradigm change, recent approaches consider both theories complementary to explain changes in communities at specific spatial scales (Legendre et al. 2009, Menezes et al. 2016).

Many biotic groups have been used to study diversity patterns at varying spatial scales. However, few ichthyofaunal comparisons of coastal environments at broader scales and many aspects that explain the relative contribution of regional processes to the community structure are unknown (Bouchon-Navarro et al. 2005). Some studies have shown that the intrinsic variability at the regional level seems to be a recurrent feature greater than the spatial differences expected depending on the scale (Sheaves 2006, Sheaves \&

Corresponding editor: Alejandra Volpedo 
Johnston 2009). However, factors such as the latitude, size, and form of an estuary, dynamics of the mouth, salinity, temperature, diversity of habitats, connectivity, and dispersal capacity can be important drivers (Vasconcelos et al. 2015).

Mangroves in the Tropical Eastern Pacific make up more than $26 \%$ of the Neotropics' total and about $8 \%$ of the world's total vegetation area (Lacerda et al. 2002). However, these areas are being lost at high rates due to increased urban, aquacultural, and agricultural development (Polidoro et al. 2013). Mangroves play a key role in many fish species' life-cycle of economic and ecological importance (Wolff 2009). Some approaches focused on characterizing near-shore fish species have included the mangrove component within the definition of soft-bottom fish. However, regional comparisons for the specific category of mangrove fish are recent (Sheaves 2012, Castellanos-Galindo et al. 2013), and there are still notable information gaps for many areas.

The Tropical Eastern Pacific region, which encompasses the American continental coast from the south of the Baja California Peninsula (Magdalena Bay) to northern Peru (Cabo Blanco), is a well-studied region in terms of its shore fish fauna (Robertson \& Allen 2015). Within this region, the Gulf of Tehuantepec, southeastern Mexico, has been pointed out as an important area whose fish fauna needs to be studied more thoroughly (Zapata \& Robertson 2007). Its environmental processes, such as upwelling, current patterns, winds, and freshwater discharges, leads to high productivity that supports artisanal and industrial fisheries. Likewise, towards its continental part, the variety of geoforms, development, and extension of its mangroves and a marked gradient of precipitation influence its fish fauna (Gómez-González et al. 2012, Romero-Berny et al. 2018). The specific aims of this study were to identify and compare spatial patterns of abundance, richness, and composition of the fish assemblages in mangrove-dominated coastal lagoon systems of the Gulf of Tehuantepec, as well as to assess the levels of non-point diversity. Here, it is hypothesized that beta diversity will make a larger contribution than alpha diversity to gamma diversity. There will be high species turnover due to high spatial heterogeneity in the Gulf of Tehuantepec region.

\section{MATERIALS AND METHODS}

\section{Study area}

The Gulf of Tehuantepec (GT) is located in front of the States of Oaxaca and Chiapas in the southeastern Mexican Pacific. Regionally, the morphotectonic unity of its littoral zone occurs from Punta Chipehua to the mouth of the Suchiate River $\left(16^{\circ} 00^{\prime}-14^{\circ} 42^{\prime} \mathrm{N}, 95^{\circ} 25^{\prime}-\right.$ $92^{\circ} 30^{\prime} \mathrm{W}$ ) (Fig. 1), although the influence of its biotic processes probably extends to Puerto San José, Guatemala (Carranza-Edwards et al. 1975, Sætersdal et al. 1999, Wilkinson et al. 2009). The region has about $420 \mathrm{~km}$ of shoreline, highlighting the coastal lagoons as the most representative geoforms (De la Lanza-Espino et al. 2013). These are shallow coastal lagoons sheltered by sandbars (Lankford 1977). The tides are mixed, predominantly semidiurnal, with a mean range of $1 \mathrm{~m}$. This region experiences a sub-humid tropical climate characterized by two distinct seasons (rainy and dry). The magnitude of annual precipitation increases northwest to southeast from below $900 \mathrm{~mm}$ to over $1600 \mathrm{~mm}$ (García 2004). The continental coastal portion has two differentiable regions: the northern, with sandy bars and alloctone basins that lead to the formation of extensive lagoons (e.g. Superior-Inferior, Mar Muerto); and the southeastern, with homogeneous beaches lacking bays (De la Lanza-Espino et al. 2013) that favor the maintenance of several smaller systems with ephemeral inlets (e.g. Carretas-Pereyra, ChantutoPanzacola).

The GT mangrove's physiography is highly influenced by prevailing climatic and geomorphological conditions. In the northern region, a narrow belt of mangroves borders the extensive lagoons, where dry conditions and hypersalinity lead to shrubby stands (dominated by Avicennia germinans and Conocarpus erectus) with low structural development (basal area $<10 \mathrm{~m}^{2} \mathrm{ha}^{-1}$; height $<8 \mathrm{~m}$ ). In contrast to the southeast, the extensive drainage basin and amplitude of the coastal floodplain allow the development of riverine forests (dominated by Rhizophora mangle and Laguncularia racemosa) with high complexity (basal area $>40 \mathrm{~m}^{2}$ ha $^{-1}$; height $>20 \mathrm{~m}$ ) (Tovilla-Hernández \& Romero-Berny 2012).

\section{Data collection}

The fish were captured between 2004 and 2016 in four coastal lagoon systems: La Joya-Buenavista (LJB; 2013-2016), Los Patos-Sólo Dios (PSD; 2014-2015), Carretas-Pereyra (CPE; 2004-2005), and ChantutoPanzacola (CHP; 2006-2007) (Fig. 1). The samplings were carried out in 63 sites located in the four systems. The collections were diurnal and included an annual cycle, monthly for CPE and CHP, and every two months for LJB and PSD, representing the rainy (from May to October) and dry (from November to April) seasons.

Fishing gear was monofilament cast nets $(4 \mathrm{~m}$ in diameter and $1.27 \mathrm{~cm}$ mesh) because these could be used at all sites and in different habitats. The standard effort was ten cast net deployments per site, after empi- 

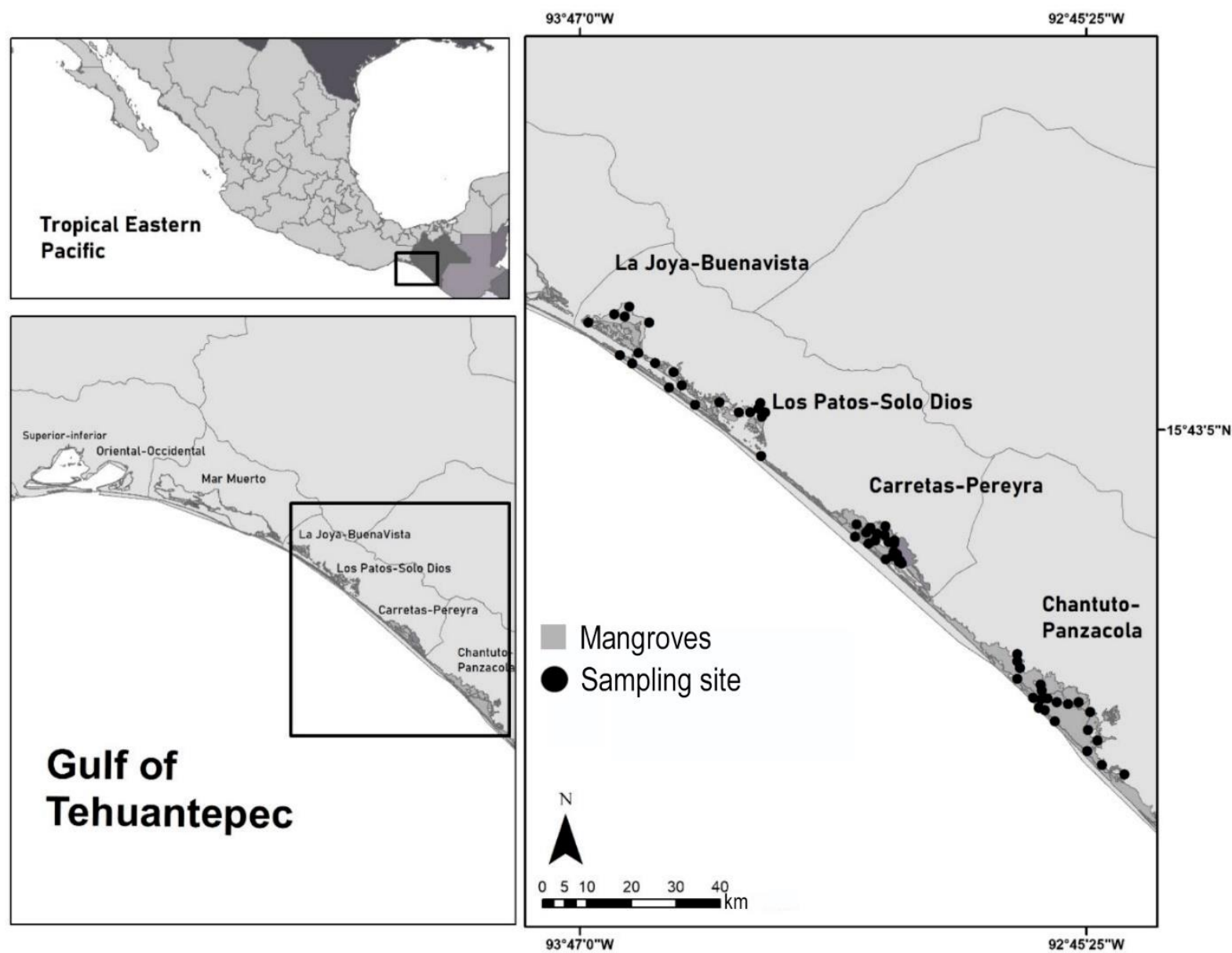

Figure 1. Mangrove-coastal lagoons in the Gulf of Tehuantepec, Mexico (Tropical Eastern Pacific).

rically verifying that this number was enough for the species accumulation curve to reach an asymptote. In each sampling, the distance to the nearest mangrove stand, depth, salinity, dissolved oxygen, $\mathrm{pH}$, and temperature were measured in the mid-depth. A different measuring equipment was used in each system: Checkmate II-S, Hach 5 (CPE), Ysi 55-DO (CHP), and Ysi 556 MPS (LJB and PSD). The fish collected were fixed in $10 \%$ formalin and transferred to the laboratory for identification with appropriate taxonomic keys (Fischer et al. 1995, Castro-Aguirre et al. 1999, Miller et al. 2009); all scientific names were updated according to Eschmeyer et al. (2017). Voucher specimens were deposited at the ichthyological collection of the Museum of Zoology of the Universidad de Ciencias y Artes de Chiapas in Tuxtla Gutiérrez, México (MZ-P-UNICACH). Species were allocated to five ecogeographical affinities according to their salinity tolerance (Myers 1949, Yáñez-Arancibia et al. 1985, Torres-Orozco 1994): primary freshwater (species found strictly in freshwater systems and are physiologically intolerant to salinity), secondary freshwater (species frequently found in freshwater systems but with some tolerance for estuarine conditions and dispersal capacity through marine environments), estuarine resident (species that are residents in brackish waters, salinity 5-10, but with tolerance to abrupt changes in salinity), marine euryhaline (species that usually tolerate salinities from 10 to 18 ) and marine stenohaline (species that usually tolerate salinities from 18 to 30 ). Fish species were also allocated to trophic guilds considering information on food items from Froese \& Pauly (2017) and other studies in the eastern Pacific (Gómez-González et al. 2012, Robertson \& Allen 2015, Romero-Berny et al. 2018). Six trophic guilds were identified: herbivores, detritivores, omnivores, zooplanktivores, zoobenthivores, and piscivores.

\section{Local-scale analysis}

The fish abundance data were fourth-root transformed to minimize the overly abundant taxa influence (Legendre \& Legendre 2012). Abundance distribution diagrams made the assemblages' general description of four systems (LJB, PSD, CPE, CHP). The interdependence of species richness and abundance with 
environmental parameters (salinity, $\mathrm{pH}$, dissolved oxygen, water temperature, and depth) and distance to the mangrove was explored for each system by a test for rank correlation coefficients.

The degree of specific dominance (dominant, common, occasional, rare) was determined through an Olmstead-Tukey test for association (Sokal \& Rohlf 1995). The abundance values were rarefied to compensate for differences in sampling effort between systems (Gotelli \& Colwell 2011). The expected species richness $\left[\mathrm{E}\left(s_{\mathrm{n}}\right)\right]$ was compared by one-way analysis of variance (ANOVA) with Welch's correction, after verifying the assumptions of normality and homogeneity of variance. The differences of abundance between systems, seasons (dry and rainy) and their interactions were analyzed with a permutational multivariate analysis of variance (PERMANOVA; Anderson 2001), on a Bray-Curtis matrix, after evaluating the homogeneity of the multivariate dispersion (PERMDISP, 9999 permutations; Anderson 2006) by factor. The seasonal factor was included in this analysis through the rarefied abundances for the groups of dry and rainy months. The multivariate patterns and the influence of the species abundance on spatial gradients were examined using Spearman correlations ( $>0.4)$ with the first two axes of a canonical analysis of principal coordinates (CAP; Anderson \& Willis 2003).

\section{Regional-scale analysis}

The degree of ichthyofaunal similarity between seven lagoon systems of the GT (Superior-Inferior, OrientalOccidental, and Mar Muerto in addition to LJB, PSD, CPE, and CHP) was examined with the Jaccard index on a matrix of presence/absence of species based on the information described here and in the inventories published by Chávez (1979), Tapia-García et al. (1998), Tapia-García \& Mendoza-Rodríguez (2005) and Gómez-González et al. (2012). The classification was by the UPGMA method, and the identification of groups in the dendrogram was performed with a test of similarity profiles (SIMPROF, 1000 permutations, and 999 simulations) at a significance level of $\alpha=0.05$. The relationship of the species classified according to their ecological affinity with the groups was determined by a Chi-square test with Monte Carlo simulations (9999 permutations), under the assumption that there were differences in the contribution of peripheral and secondary species between each group (Avilés-Torres et al. 2001).

The rate of spatial replacement (beta diversity) between systems was measured with the Cody index, representing the number of species gained or lost when moving between systems (Magurran 1988). The index was estimated using the following formula: $\mathrm{BCI}=$ $[\mathrm{g}(\mathrm{H})+\mathrm{I}(\mathrm{H})] / 2$, where BCI: Cody index, $\mathrm{g}(\mathrm{H})$ : number of species present in system 1 but absent in system $2, \mathrm{I}(\mathrm{H})$ : number of species absent in system 1 but present in system 2. The obtained values were employed to conduct a triangular matrix, which was then used to create a Cluster analysis (UPGMA) and a non-metric multidimensional scaling plot (nMDS). The ordination was correlated with salinity's mean values and each system's mangrove area using Spearman coefficients. The salinity values for the Mar Muerto, Superior-Inferior, and Oriental-Occidental systems were taken from Chávez et al. (1977), Castro-Aguirre (1982), and Contreras et al. (1997). The mangrove areas for each system were taken from Tovilla-Hernández et al. $(2007,2011)$ (Table 1).

The landscape heterogeneity was estimated from the existing species diversity in its spatial dimension, expressed as gamma (Schluter \& Ricklefs 1993) with the following formula: $\Gamma=\bar{\alpha} \times \beta \times \mathrm{N}$, where $\Gamma$ : gamma diversity, $\bar{\alpha}$ : mean number of species, $\beta$ : the inverse of species dimension; expressed as $1 /$ the mean presence of systems occupied by a species. $\mathrm{N}$ : total number of systems.

Statistical analyses were carried out in PERMANOVA+ for PRIMER 6 (Anderson et al. 2008) and PAST 3.16 (Hammer et al. 2001).

\section{RESULTS}

\section{Local-scale analysis}

The fish fauna for the four sampled mangrove-systems comprised 89 species (Table 2) in 31 families. The most specious families were Carangidae (seven species), Engraulidae, Ariidae, Gerreidae, and Haemulidae (each with six species). Other important families were Poeciliidae, Centropomidae, Sciaenidae, and Gobiidae (with five species each). The abundance range plots visually show a log-normal distribution shape, which indicates a few very abundant species and many rare ones (Fig. 2). In terms of their mean relative abundance, the most common species in these systems was Lile gracilis (Clupeidae, $\bar{X}=13.6 \%$ ); other important species in this component were Poecilia nelsoni (Poeciliidae, $\bar{X}=8.9 \%$ ), Dormitator latifrons (Eleotridae, $\bar{X}=8.5 \%$ ) and Centropomus robalito (Centropomidae, $\bar{X}=8.4 \%)$. However, the general pattern of abundance varied considerably in CHP, where the most abundant species were Astyanax aeneus (Characidae, 8.7\%) and Anchoa mundeola (Engraulidae, 7.5\%).

According to Olmstead-Tukey analysis, 59.3\% of species were classified as rare, $20.9 \%$ as dominant, $17.4 \%$ as common, and only $2.3 \%$ as occasional. 
Table 1. Mangrove area and average salinity values for seven mangrove-coastal lagoon systems of the Gulf of Tehuantepec, Mexico (Tropical Eastern Pacific). Salinity values for Superior-Inferior, Oriental-Occidental, and Mar Muerto were taken from Chávez et al. (1977), Castro-Aguirre (1982), and Contreras et al. (1997). Mangrove area values were taken from Tovilla et al. $(2007,2012)$.

\begin{tabular}{lccccccc}
\hline & $\begin{array}{c}\text { Superior- } \\
\text { Inferior }\end{array}$ & $\begin{array}{c}\text { Oriental- } \\
\text { Occidental }\end{array}$ & $\begin{array}{c}\text { Mar } \\
\text { Muerto }\end{array}$ & $\begin{array}{c}\text { La Joya- } \\
\text { Buenavista }\end{array}$ & $\begin{array}{c}\text { Los Patos- } \\
\text { Solo Dios }\end{array}$ & $\begin{array}{c}\text { Carretas- } \\
\text { Pereyra }\end{array}$ & $\begin{array}{c}\text { Chantuto- } \\
\text { Panzacola }\end{array}$ \\
\hline Mangrove area $\left(\mathrm{km}^{2}\right)$ & 17.8 & 15.1 & 110.04 & 25.31 & 63.74 & 81.21 & 274.77 \\
Salinity & 30 & 39 & 35 & 18 & 19 & 11 & 12 \\
\hline
\end{tabular}

Table 2. Relative abundance (A\%) and frequency of occurrence (F\%) of fish species collected between 2004 and 2016 at four mangrove-coastal lagoon systems of the Gulf of Tehuantepec, Mexico (Tropical Eastern Pacific). Species are sorted according to decreasing mean abundance. EA: ecological affinity, ER: estuarine resident, EU: euryhaline, ST: stenohaline, PF: primary freshwater, SF: secondary freshwater, C: catadromous. TG: trophic group, DV: detritivore, ZP: zooplanktivore, ZB: zoobenthivore, PV: piscivore, OV: omnivore, HV: herbivore. Relative values based on the total number of specimens and number of sampling events per system: LBJ: La Joya-Buenavista, 2064 specimens, 78 samplings; PSD: Los Patos-Solo Dios, 1215 specimens, 48 samplings; CPE: Carretas-Pereyra, 11797 specimens, 264 samplings; CHP: Chantuto-Panzacola, 3941 specimens, 240 samplings. (-) Refers to species absent at the sample, *Exotic species.

\begin{tabular}{|c|c|c|c|c|c|c|c|c|c|c|}
\hline \multirow{3}{*}{ Species } & \multirow{3}{*}{ EA } & \multirow{3}{*}{ TG } & \multicolumn{8}{|c|}{ Mangrove-coastal lagoon systems } \\
\hline & & & \multicolumn{2}{|c|}{ LJB } & \multicolumn{2}{|c|}{ PSD } & \multicolumn{2}{|c|}{$\mathrm{CPE}$} & \multicolumn{2}{|c|}{$\mathrm{CHP}$} \\
\hline & & & $\mathrm{A} \%$ & $\mathrm{~F} \%$ & $\mathrm{~A} \%$ & $\mathrm{~F} \%$ & $\mathrm{~A} \%$ & $\mathrm{~F} \%$ & $\mathrm{~A} \%$ & $\mathrm{~F} \%$ \\
\hline Lile gracilis & ER & $\mathrm{ZP}$ & 13.91 & 6.42 & 20.41 & 4.76 & 16.65 & 6.46 & 3.32 & 3.87 \\
\hline Poecilia nelsoni & SF & $\mathrm{ZB}$ & 9.69 & 3.21 & 10.45 & 3.47 & 14.55 & 3.69 & 1.04 & 1.49 \\
\hline Dormitator latifrons & ER & DV & 5.57 & 2.67 & 7.65 & 4.76 & 14.08 & 4.62 & 6.72 & 2.98 \\
\hline Centropomus robalito & $\mathrm{EU}$ & PV & 5.86 & 4.28 & 16.87 & 5.56 & 4.82 & 5.85 & 6.11 & 3.87 \\
\hline Astatheros macracanthus & SF & OV & 10.08 & 5.88 & 12.02 & 5.56 & 4.20 & 4.92 & 3.45 & 2.08 \\
\hline Poecilia sphenops & SF & $\mathrm{ZB}$ & 3.63 & 1.07 & 0.99 & 3.97 & 14.72 & 2.15 & 0.89 & 1.79 \\
\hline Diapterus brevirostris & $\mathrm{EU}$ & $\mathrm{OV}$ & 5.47 & 6.42 & 3.29 & 3.17 & 3.48 & 5.54 & 6.09 & 4.46 \\
\hline Atherinella guatemalensis & ER & ZB & 3.05 & 4.28 & 1.07 & 3.97 & 6.65 & 4.62 & 5.66 & 1.49 \\
\hline Eucinostomus currani & $\mathrm{EU}$ & OV & 9.98 & 4.81 & 1.65 & 1.59 & 0.53 & 3.08 & 4.19 & 2.98 \\
\hline Mugil curema & $\mathrm{EU}$ & DV & 6.25 & 4.28 & 1.15 & 3.17 & 2.24 & 4.62 & 3.75 & 5.36 \\
\hline Astyanax aeneus & $\mathrm{PF}$ & $\mathrm{OV}$ & 0.05 & 0.53 & 1.89 & 2.38 & 2.45 & 3.69 & 8.70 & 2.38 \\
\hline Anchoa mundeola & EU & $\mathrm{ZP}$ & 1.26 & 3.74 & 3.70 & 6.35 & - & - & 7.46 & 3.57 \\
\hline Amphilophus trimaculatus & SF & $\mathrm{ZB}$ & 3.92 & 3.21 & 3.37 & 3.97 & 2.39 & 4.92 & 2.66 & 2.08 \\
\hline Gobiomorus maculatus & ER & PV & 1.14 & 1.07 & 1.65 & 4.76 & 2.48 & 5.54 & 4.62 & 3.57 \\
\hline Ariopsis guatemalensis & EU & $\mathrm{ZB}$ & 0.73 & 1.60 & 0.49 & 1.59 & 1.20 & 4.31 & 6.75 & 4.76 \\
\hline Gobionellus microdon & ER & DV & 1.11 & 3.21 & 2.47 & 3.97 & 0.68 & 5.54 & 3.50 & 2.68 \\
\hline Poeciliopsis fasciata & SF & DV & 0.68 & 1.60 & 1.73 & 2.38 & 3.79 & 3.38 & - & - \\
\hline Cathorops liropus & $\mathrm{EU}$ & $\mathrm{ZB}$ & 0.15 & 1.07 & 0.49 & 1.59 & - & - & 4.67 & 4.76 \\
\hline Gerres simillimus & $\mathrm{EU}$ & OV & 3.97 & 5.35 & 0.33 & 1.59 & 0.3 & 0.62 & 0.18 & 1.49 \\
\hline Anchoa lucida & EU & $\mathrm{ZP}$ & 0.73 & 2.14 & 0.16 & 1.59 & 1.35 & 4.62 & 2.36 & 2.08 \\
\hline Caranx caninus & $\mathrm{EU}$ & $\mathrm{ZB}$ & 0.48 & 2.14 & 0.08 & 0.79 & 0.10 & 0.92 & 3.83 & 3.87 \\
\hline Anchovia macrolepidota & $\mathrm{ES}$ & $\mathrm{ZP}$ & 0.73 & 0.53 & 1.40 & 3.17 & 0.65 & 2.15 & 1.47 & 1.79 \\
\hline Lutjanus argentiventris & ES & PV & 0.68 & 1.60 & 0.16 & 1.59 & 0.05 & 0.92 & 2.59 & 2.08 \\
\hline Poeciliopsis turrubarensis & SF & DV & - & - & 2.72 & 2.38 & 0.29 & 0.31 & 0.23 & 0.30 \\
\hline Lile nigrofasciata & ER & $\mathrm{ZP}$ & 0.58 & 1.07 & - & - & 0.62 & 2.46 & 1.34 & 1.79 \\
\hline Opisthonema libertate & ES & $\mathrm{ZP}$ & 2.28 & 1.07 & - & - & - & - & - & - \\
\hline Citharichthys gilberti & EU & PV & 0.34 & 1.07 & - & - & 0.19 & 0.62 & 1.52 & 1.79 \\
\hline Oligoplites altus & EU & $\mathrm{ZB}$ & 0.78 & 2.67 & 0.16 & 1.59 & 0.14 & 1.23 & 0.94 & 4.17 \\
\hline Achirus mazatlanus & EU & $\mathrm{ZB}$ & 0.15 & 1.07 & 0.82 & 3.97 & 0.50 & 2.77 & 0.53 & 2.68 \\
\hline Cathorops fuerthii & EU & $\mathrm{ZB}$ & - & - & - & - & 0.59 & 2.15 & 1.32 & 4.65 \\
\hline Membras gilberti & $\mathrm{ES}$ & $\mathrm{ZP}$ & 1.31 & 1.07 & - & - & - & - & 0.51 & 0.30 \\
\hline Anableps dowii & ER & $\mathrm{OV}$ & 0.15 & 0.53 & 1.07 & 3.17 & - & - & 0.20 & 0.60 \\
\hline Centropomus nigriscens & EU & PV & 0.73 & 2.14 & 0.33 & 2.38 & 0.04 & 0.92 & 0.10 & 0.89 \\
\hline Cathorops steindachneri & EU & $\mathrm{ZB}$ & 0.19 & 1.07 & 0.49 & 1.59 & - & - & 0.33 & 0.89 \\
\hline Centropomus armatus & EU & PV & 0.97 & 1.60 & - & - & - & - & - & - \\
\hline
\end{tabular}


continuation

\begin{tabular}{|c|c|c|c|c|c|c|c|c|c|c|}
\hline \multirow{3}{*}{ Species } & \multirow{3}{*}{ EA } & \multirow{3}{*}{ TG } & \multicolumn{8}{|c|}{ Mangrove-coastal lagoon systems } \\
\hline & & & \multicolumn{2}{|c|}{ LJB } & \multicolumn{2}{|c|}{ PSD } & \multicolumn{2}{|c|}{$\mathrm{CPE}$} & \multicolumn{2}{|c|}{ CHP } \\
\hline & & & $\mathrm{A} \%$ & $\mathrm{~F} \%$ & $\mathrm{~A} \%$ & $\mathrm{~F} \%$ & $\mathrm{~A} \%$ & $\mathrm{~F} \%$ & $\mathrm{~A} \%$ & $\mathrm{~F} \%$ \\
\hline Oligoplites saurus & $\mathrm{EU}$ & $\mathrm{ZP}$ & 0.19 & 1.07 & 0.16 & 0.59 & 0.08 & 1.54 & 0.51 & 2.08 \\
\hline Notarius kessleri & EU & $\mathrm{ZB}$ & - & - & - & - & - & - & 0.76 & 0.70 \\
\hline Eugerres lineatus & ES & OV & 0.44 & 2.67 & 0.25 & 2.38 & - & - & - & - \\
\hline Poeciliopsis pleurospilus & SF & DV & 0.44 & 0.53 & 0.16 & 1.59 & - & - & - & - \\
\hline Roeboides bouchellei & $\mathrm{PF}$ & $\mathrm{ZB}$ & - & - & - & - & 0.02 & 0.31 & 0.46 & 0.60 \\
\hline Lutjanus novemfasciatus & EU & PV & 0.48 & 2.14 & - & - & - & - & - & - \\
\hline Eugerres axillaris & $\mathrm{ES}$ & OV & 0.44 & 2.14 & - & - & - & - & - & - \\
\hline Centropomus medius & ES & PV & - & - & - & - & 0.03 & 0.62 & 0.38 & 1.19 \\
\hline Centropomus viridis & $\mathrm{EU}$ & PV & 0.24 & 1.07 & 0.08 & 0.79 & - & - & 0.08 & 0.60 \\
\hline Eucinostomus dowii & $\mathrm{EU}$ & ZB & - & - & - & - & - & - & 0.30 & 0.60 \\
\hline Hyporhamphus naos & EU & $\mathrm{ZP}$ & 0.24 & 1.07 & - & - & - & - & 0.03 & 0.30 \\
\hline Sphoeroides annulatus & EU & $\mathrm{ZB}$ & 0.15 & 1.07 & - & - & 0.01 & 0.31 & 0.10 & 1.19 \\
\hline Mugil hospes & EU & DV & 0.10 & 0.53 & - & - & 0.02 & 0.62 & 0.10 & 0.30 \\
\hline Eleotris picta & ER & PV & - & - & 0.08 & 0.79 & - & - & 0.13 & 0.89 \\
\hline Selene brevoorti & $\mathrm{ES}$ & PV & - & - & - & - & - & - & 0.20 & 0.89 \\
\hline Mugil cephalus & EU & DV & 0.10 & 1.07 & - & - & 0.04 & 0.92 & 0.03 & 0.30 \\
\hline Lutjanus colorado & $\mathrm{ES}$ & PV & 0.10 & 1.07 & - & - & - & - & 0.05 & 0.60 \\
\hline Anchoa starksi & EU & $\mathrm{ZP}$ & - & - & 0.08 & 0.79 & - & - & 0.05 & 0.30 \\
\hline Aboma etheostoma & $\mathrm{EU}$ & $\mathrm{ZB}$ & 0.10 & 0.53 & - & - & - & - & 0.03 & 0.30 \\
\hline Anchoa ischana & EU & $\mathrm{ZP}$ & - & - & 0.08 & 0.79 & - & - & 0.03 & 0.30 \\
\hline Pomadasys macracanthus & $\mathrm{ES}$ & ZB & - & - & - & - & 0.01 & 0.31 & 0.10 & 0.89 \\
\hline Elops affinis & ES & PV & - & - & - & - & 0.02 & 0.31 & 0.08 & 0.60 \\
\hline Ariopsis seemani & $\mathrm{EU}$ & $\mathrm{ZB}$ & - & - & - & - & 0.08 & 0.23 & - & - \\
\hline Trinectes fonsecensis & EU & ZB & - & - & - & - & - & - & 0.08 & 0.30 \\
\hline Caranx sexfasciatus & $\mathrm{ES}$ & PV & - & - & - & - & - & - & 0.08 & 0.30 \\
\hline Stellifer wintersteenorum & EU & $\mathrm{ZB}$ & - & - & - & - & - & - & 0.08 & 0.30 \\
\hline Haemulopsis axillaris & $\mathrm{ES}$ & ZB & 0.05 & 0.53 & - & - & - & - & - & - \\
\hline Orthopristis chalceus & ES & $\mathrm{ZB}$ & 0.05 & 0.53 & - & - & - & - & - & - \\
\hline Rhamdia guatemalensis & $\mathrm{PF}$ & ZB & - & - & - & - & - & - & 0.05 & 0.60 \\
\hline Halichoeres dispilus & $\mathrm{ES}$ & ZB & 0.05 & 0.53 & - & - & - & - & - & - \\
\hline Cynoscion albus & $\mathrm{EU}$ & PV & - & - & - & - & - & - & 0.05 & 0.60 \\
\hline Bairdiella armata & $\mathrm{ES}$ & $\mathrm{ZB}$ & - & - & - & - & - & - & 0.05 & 0.30 \\
\hline Oreochromis niloticus* & $\mathrm{SF}$ & HV & - & - & - & - & 0.01 & 0.31 & 0.03 & 0.30 \\
\hline Anchoa curta & $\mathrm{EU}$ & $\mathrm{ZP}$ & - & - & - & - & 0.03 & 0.31 & - & - \\
\hline Pomadasys bayanus & ES & ZB & - & - & - & - & - & - & 0.03 & 0.30 \\
\hline Polydactylus approximans & $\mathrm{ES}$ & $\mathrm{ZB}$ & - & - & - & - & - & - & 0.03 & 0.30 \\
\hline Kyphosus elegans & $\mathrm{ES}$ & HV & - & - & - & - & - & - & 0.03 & 0.30 \\
\hline Atractosteus tropicus & SF & PV & - & - & - & - & - & - & 0.03 & 0.30 \\
\hline Hemicaranx zelotes & $\mathrm{ES}$ & PV & - & - & - & - & - & - & 0.03 & 0.30 \\
\hline Synodus scituliceps & ES & PV & - & - & - & - & - & - & 0.03 & 0.30 \\
\hline Micropogonias altipinnis & $\mathrm{EU}$ & ZB & - & - & - & - & - & - & 0.03 & 0.30 \\
\hline Hyporhamphus snyderi & $\mathrm{EU}$ & $\mathrm{ZP}$ & - & - & - & - & - & - & 0.03 & 0.30 \\
\hline Hippocampus ingens & $\mathrm{ES}$ & $\mathrm{ZP}$ & - & - & - & - & - & - & 0.03 & 0.30 \\
\hline Haemulopsis leuciscus & ES & $\mathrm{ZB}$ & - & - & - & - & - & - & 0.03 & 0.30 \\
\hline Ctenogobius sagittula & $\mathrm{EU}$ & DV & - & - & - & - & - & - & 0.03 & 0.30 \\
\hline Chaetodon humeralis & ES & OV & - & - & - & - & - & - & 0.03 & 0.30 \\
\hline Genyatremus pacifici & ES & ZB & - & - & - & - & - & - & 0.03 & 0.30 \\
\hline Microgobius miraflorensis & ER & DV & - & - & - & - & - & - & 0.03 & 0.30 \\
\hline Rhinoptera steindachneri & ES & ZB & - & - & - & - & - & - & 0.03 & 0.30 \\
\hline Trachinotus kennedyi & $\mathrm{ES}$ & PV & - & - & - & - & - & - & 0.03 & 0.30 \\
\hline Lutjanus guttatus & ES & PV & - & - & - & - & - & - & 0.03 & 0.30 \\
\hline Bairdiella ensifera & $\mathrm{ES}$ & $\mathrm{ZB}$ & - & - & - & - & - & - & 0.03 & 0.30 \\
\hline Sphoeroides rosenblatti & EU & $\mathrm{ZB}$ & - & - & - & - & - & - & 0.03 & 0.30 \\
\hline Awaous banana & $\mathrm{C}$ & DV & - & - & - & - & 0.01 & 0.31 & - & - \\
\hline
\end{tabular}


LA JOYA-BUENAVISTA

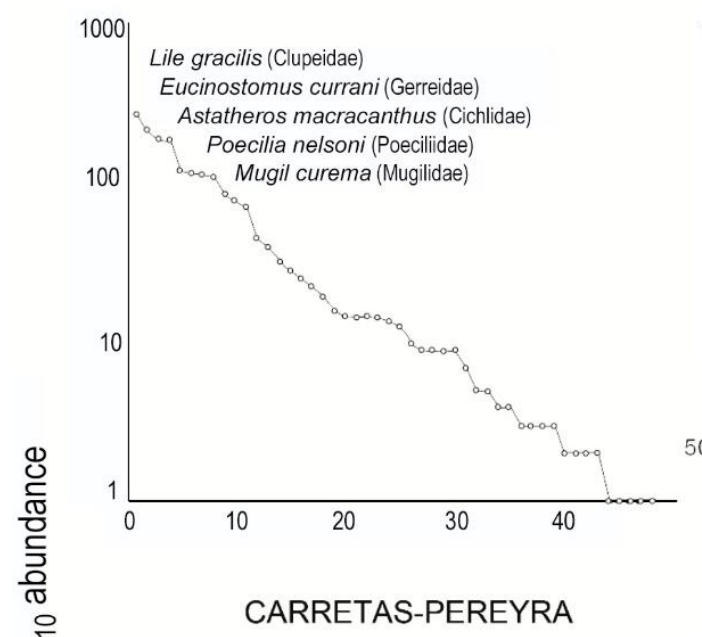

LOS PATOS-SOLO DIOS

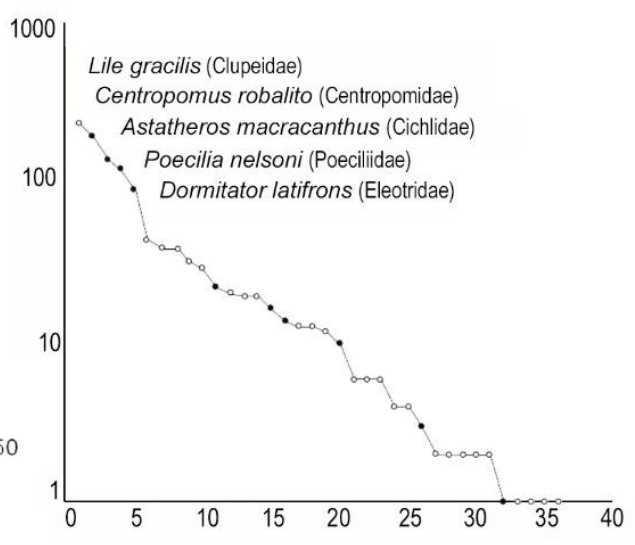

CHANTUTO-PANZACOLA

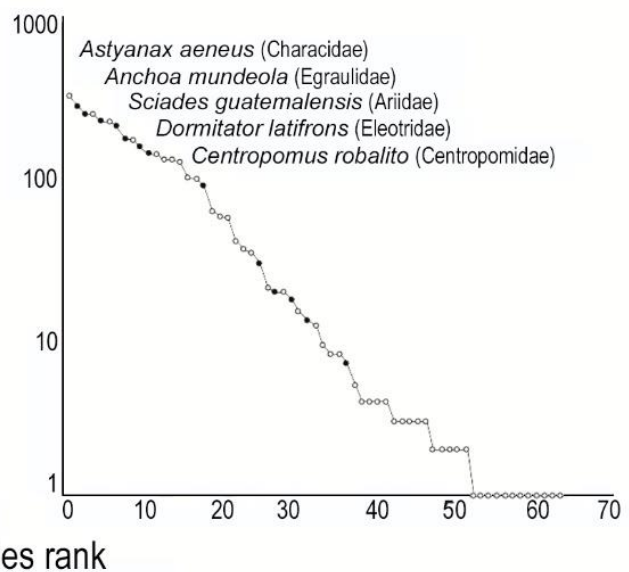

Figure 2. Rank-abundance $\left(\log _{10}\right)$ plots for fish assemblages in four mangrove-coastal lagoon systems of the Gulf of Tehuantepec (Tropical Eastern Pacific). The five most abundant species are provided for each system.

Among systems, the number of species was variable, with the highest values for CHP $\left(s_{\mathrm{obs}}=75, \mathrm{E} s_{1204}=52\right)$ and the lowest for PSD $\left(s_{\mathrm{obs}}=36, \mathrm{E} s_{1204}=29\right)$, with significant differences in the expected richness $(F=$ 2.75, $P<0.0001)$. The PERMANOVA test results showed significant differences in the abundance between the four systems and between the seasons (rainy and dry); it also indicated a significant system $\times$ season interaction (Table 3), and the ordination plot reflected the spatial pattern (Fig. 3). The CAP ordering was significant $\left(\delta^{2}=0.69, P=0.0001\right)$, with a total classification success of $74.6 \%$, indicating spatial separation, grouping the contiguous systems (Fig. 3). Astatheros macracanthus ( $r=0.59)$, Gerres simillimus $(r=0.57)$ and Eugerres lineatus $(r=0.42)$ abundances were moderately correlated with CAP axis 1 , whereas Caranx caninus $(r=0.56)$, Cathorops steindachneri $(r$ $=0.47)$ and Poeciliopsis fasciata $(r=-0.47)$ were moderately correlated with the CAP axis 2 .
Table 3. Results of PERMANOVA comparing fish assemblages between systems and seasons. df: degrees of freedom, ss: the sum of squares, ms: mean squares, $F$ : pseudo-F statistic value. *Significant $P$ values $(<0.05)$.

\begin{tabular}{lccccc}
\hline Source & df & ss & ms & $F$ & $P$ \\
\hline System & 3 & 11482 & 3827.3 & 4.956 & $0.0001^{*}$ \\
Season & 1 & 1925.2 & 1925.2 & 2.296 & $0.0166^{*}$ \\
System $\times$ Season & 3 & 3887.3 & 1295.8 & 1.678 & $0.0125^{*}$ \\
Residual & 16 & 12356 & 772.28 & & \\
Total & 34 & 41613 & & & \\
\hline
\end{tabular}

Dissolved oxygen, distance to mangroves, and depth showed a significant correlation $(P<0.05)$ with any of the components (richness or abundance) of the fish assemblages for one or more systems. However, the regression coefficient values $(r<0.75)$ were relatively low (Table 4$)$.

Due to their ecogeographical and trophic affinities, the functional groups of freshwater and zoobenthivorous 


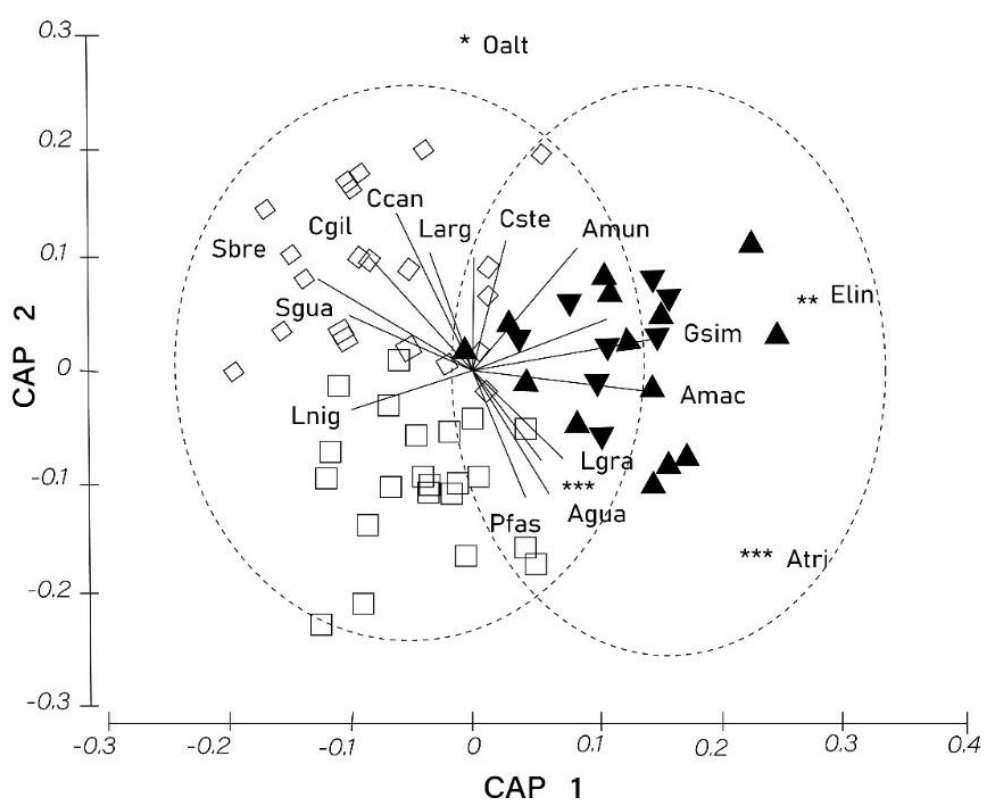

Figure 3. Canonical analysis of principal coordinates biplot indicating spatial patterns of fish assemblages in four mangrove-coastal lagoon systems of the Gulf of Tehuantepec (Tropical Eastern Pacific). Vectors of species based on Spearman correlation values >0.4. Abbreviations for species name: Ccan: Caranx caninus, Larg: Lutjanus argentiventris, *Oalt: Oligoplites altus, Cste: Cathorops steindachneri, Amun: Anchoa mundeola, **Elin: Eugerres lineatus, Gsim: Gerres simillimus, Amac: Asthateros macracanthus, Lgra: Lile gracilis, ***Agua: Atherinella guatemalensis, Pfas: Poeciliopsis fasciata, Lnig: Lile nigrofasciata, Sgua: Sciades guatemalensis, Sbre: Selene brevoortii, Cgil: Citharichthys gilberti. Dotted ellipses indicate groups of lagoons and symbols indicate the sampling sites. $\mathbf{\Delta}$ : LJB, $\nabla$ : PSD, $\square$ : CPE, $\diamond:$ CHP.

Table 4. Spearman range coefficients between mean values \pm standard deviation of environmental variables for fish samples and species richness and abundance in four systems in the Gulf of Tehuantepec, Mexico (Tropical Eastern Pacific). LJB: La Joya-Buenavista, PSD: Los Patos-Solo Dios, CPE: Carretas-Pereyra, CHP: Chantuto-Panzacola. S: species richness, A: abundance. *Permutation test significant values $(P<0.05)$.

\begin{tabular}{|c|c|c|c|c|c|c|}
\hline Environmental variables & \multicolumn{6}{|c|}{ Mangrove-coastal lagoon systems } \\
\hline \multirow{3}{*}{ Salinity } & \multirow{2}{*}{$\frac{\text { LJB }}{17.7 \pm 6.4}$} & \multirow{2}{*}{$\frac{\text { PSD }}{18.9 \pm 3}$} & \multirow{2}{*}{\multicolumn{2}{|c|}{$\frac{\text { CPE }}{10.9 \pm 9.8}$}} & \multicolumn{2}{|c|}{ CHP } \\
\hline & & & & & $12 \pm$ & \pm 8.1 \\
\hline & $\mathrm{S} \quad \mathrm{A}$ & S A & $\mathrm{S}$ & A & & A \\
\hline & -0.060 .11 & $0.38 \quad 0.12$ & 0.05 & -0.13 & 0.10 & 0.12 \\
\hline \multirow[t]{3}{*}{ Dissolved oxygen $\left(\mathrm{mg} \mathrm{L}^{-1}\right)$} & $4.8 \pm 1.3$ & $4 \pm 1.8$ & 3.4 & \pm 1 & $3.7 \pm$ & \pm 1.3 \\
\hline & $\mathrm{S} \quad \mathrm{A}$ & A & $\mathrm{S}$ & A & & A \\
\hline & $-0.01-0.39 *$ & $0.30 \quad 0.09$ & $-0.27 *$ & $-0.25^{*}$ & -0.12 & $-0.15^{*}$ \\
\hline \multirow[t]{3}{*}{$\mathrm{pH}$} & $7.9 \pm 0.4$ & $7.7 \pm 0.4$ & $7.5 \pm$ & \pm 0.5 & $7.4 \pm$ & \pm 0.3 \\
\hline & $\mathrm{S} \quad \mathrm{A}$ & S A & $\mathrm{S}$ & A & & A \\
\hline & $-0.08-0.26$ & 0.180 .10 & -0.14 & -0.14 & 0.07 & 0.09 \\
\hline \multirow[t]{3}{*}{ Water temperature $\left({ }^{\circ} \mathrm{C}\right)$} & $30.9 \pm 0.7$ & $30.7 \pm 0.8$ & 31.1 & \pm 1.8 & $30 \pm$ & \pm 1.3 \\
\hline & S A & $\mathrm{S} \quad \mathrm{A}$ & $\mathrm{S}$ & A & & A \\
\hline & $-0.49-0.51$ & 0.380 .11 & 0.22 & -0.10 & -0.01 & 0.02 \\
\hline \multirow[t]{3}{*}{ Depth (m) } & $0.8 \pm 0.2$ & $1.3 \pm 0.6$ & $1.1 \pm$ & \pm 0.9 & $1.3 \pm$ & \pm 1.2 \\
\hline & $\mathrm{S} \quad \mathrm{A}$ & S A & $\mathrm{S}$ & A & & A \\
\hline & -0.160 .11 & $0.74 * 0.15$ & 0.02 & 0.34 & -0.04 & -0.03 \\
\hline \multirow[t]{3}{*}{ Distance to mangrove (m) } & $6.7 \pm 2.2$ & $5.8 \pm 2.7$ & $6.5 \pm$ & \pm 2.4 & $5.4 \pm$ & \pm 2.3 \\
\hline & A & $\mathrm{S} \quad \mathrm{A}$ & $\mathrm{S}$ & A & & A \\
\hline & $0.30 \quad 0.46$ & $0.230 .53 *$ & -0.10 & $0.43 *$ & $0.75^{*}$ & $0.73 *$ \\
\hline
\end{tabular}

species were the most abundant $(\bar{X}=32.9 \%$ and $\bar{X}=$ $29.2 \%$, respectively). However, for CHP and PSD systems, euryhaline (51.4\%) and zooplanktivorous $(26.2 \%)$ species were more abundant. 


\section{Regional-scale analysis}

According to the ichthyofaunal composition of seven coastal lagoon systems along with the GT, there were two significant complexes of localities: one to the north (called here as Istmo) that included the Mar Muerto system and the extensive lagoons of the Huave system (Superior, Inferior, Oriental, Occidental), and another one towards the east (called here Soconusco), that grouped the four systems analyzed in this study (Fig. 4). In addition to this last group, CHP is separated from CPE-PSD and LJB due to its high richness. On the ecogeographical derivation, for both groups the best represented component was the marine (euryhaline $\bar{s}=$ $33 \pm 11$, stenohaline $\bar{s}=24 \pm 20$ ); however, a greater number of freshwater $(\bar{s}=12 \pm 2)$ and resident estuarine species $(\bar{s}=7 \pm 3)$ in Soconusco compared to Istmo (freshwater $\bar{s}=6 \pm 3$, resident $\bar{s}=4 \pm 1$ ), could contribute to significantly differentiate its composition $\left(\chi^{2}=40.89, P=0.002\right)$.

The replacement rate presents a gradual increase from north to south, including within the Soconusco systems. The majority of the systems gave high replacement rates compared with the $\mathrm{CHP}$, which showed a lower dissimilarity with the $\mathrm{CPE}$ contiguous system despite the richness differences. By exploring the relationship of the variables associated with the habitat, and its influence on beta diversity, the ordination plot reflected the spatial gradient, as well as a high positive correlation value (nMDS 1$)$ with the mangrove area $(\rho=0.75)$, being negative $(\rho=-0.89)$ for salinity, which was associated with the systems of Istmo (Fig. 5). The estimated value of range (gamma) diversity for the GT lagoon-estuarine region was 176 species $\left(69\right.$ species $\times 0.36$ systems $^{-1} \times 7$ systems; Table $5)$.

\section{DISCUSSION}

Although the four lagoon systems analyzed (LJB, PSD, CPE, CHP) are close to each other (166 km between the two most distant sites), the results showed consistent structural differences in their fish assemblages. The CHP system presented a high species richness, in clear contrast to the PSD. It has been suggested that spatially close local communities may be influenced by common factors (Whitfield 1999), with discontinuities between zones (Legendre \& Fortin 1989). The variations in fish assemblages between systems were mainly due to a higher abundance of species such as $L$. gracilis, $A$. macracanthus, and $P$. nelsoni, common for LJB, PSD, and CPE. In contrast, in CHP, the species composition differed markedly due to the geomorphic and physicochemical heterogeneity (Raz-Guzmán \& Huidobro 2002).

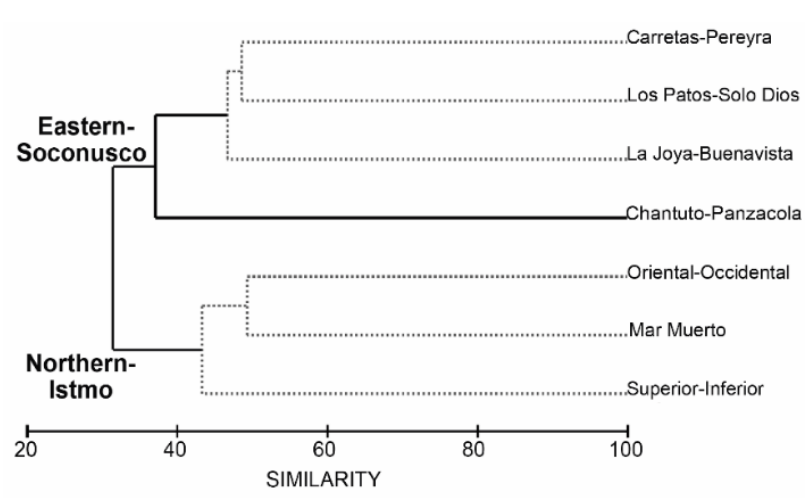

Figure 4. Dendrogram of ichthyofaunas similarity between coastal lagoon systems of the Gulf of Tehuantepec (Tropical Eastern Pacific). The dotted lines show the groups identified with a significant internal structure (SIMPROF, $P<0.05$ ). Presence/absence matrix from the lists for Carretas-Pereyra and ChantutoPanzacola (Gómez-González et al. 2013); Los Patos-Solo Dios and La Joya-Buenavista (Romero-Berny unpubl.); Oriental-Occidental (Chávez 1979); Mar Muerto (TapiaGarcía et al. 1998) and Superior-Inferior (Tapia-García \& Mendoza-Rodríguez 2005).

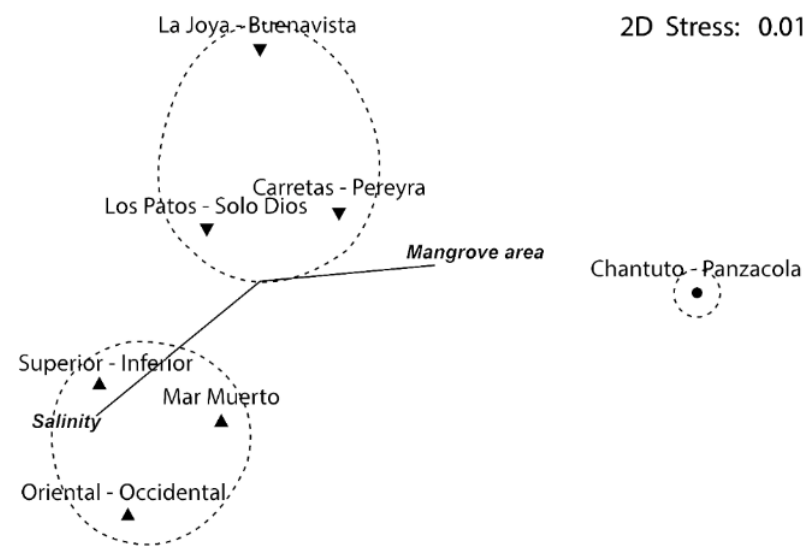

Figure 5. Ordination and classification of seven lagoon systems of the Gulf of Tehuantepec (Tropical Eastern Pacific) according to the nMDS and cluster analysis results based on the spatial turnover rate. Dashed circles encompass the systems representing the ecoregional groups defined by hierarchical clustering ( $40 \%$ similarity level). The vectors represent the Pearson correlation values between the variables and the ordination axes.

The pattern of abundance found here follows a common feature for other biological communities in tropical systems: the dominance of few species and many rare species with low abundance (Whitfield 1999, Magurran \& Henderson 2003). Concerning their ecogeographical affinity, a high proportion of resident estuarine and secondary fish was relatively common, probably reflecting the lagoons' prevailing mesohaline conditions. In CHP, depending on a relatively wide and 
Table 5. Gamma diversity values obtained for coastal lagoon systems in the Gulf of Tehuantepec and Bahía de La Paz of the Mexican Pacific (Tropical Eastern Pacific). *Values for Bahía de La Paz, Baja California Sur calculated from the data of López-Rasgado (2012).

\begin{tabular}{lcccc}
\hline & Istmo & Soconusco & Gulf of Tehuantepec & Bahía de La Paz $^{*}$ \\
\hline $\bar{\alpha}$ (Species) & 53.33 & 80.75 & 69 & 36.67 \\
$\beta$ (Systems-1) & 0.53 & 0.49 & 0.36 & 0.61 \\
$\mathrm{~N}$ (Systems) & 3 & 4 & 7 & 3 \\
$\Gamma$ (Species) & 85 & 158 & 176 & 67 \\
\hline
\end{tabular}

permanently open mouth $(640 \mathrm{~m})$, the general pattern showed a greater percentage of euryhaline species (e.g. A. mundeola, Ariopsis guatemalensis) contrasting with the other three systems, which can affect variations in salinity, dissolved oxygen, $\mathrm{pH}$, and water temperature, as well as the response of fish assemblages from a functional perspective (Díaz-Ruiz et al. 2006, Mendoza et al. 2009, López-Vila et al. 2019).

The distance to mangroves was highly correlated with fish species richness and abundance, except for LJB, where there are less mangrove coverage and complexity (Tovilla-Hernández et al. 2007). A particular ichthyofauna affinity detected between LJB and PSD, as well as between the CHP and CPE (Fig. 3), may reflect the environmental effect of contiguous localities (Whitfield 1999); but also the similarity of its mangroves (Kuo et al. 1999, Rönnbäck et al. 1999) or even management policies (Halpern 2003), since the last two systems are part of the federally protected area La Encrucijada. The dominant trophic preferences were towards species that feed on benthic invertebrates and zooplankton, resulting from these prey availability. Mangrove systems are efficient sediment sinks and significant sources of autochthonous organic matter (Prasad et al. 2010), defining environmental and productivity gradients that determine the benthic infauna distribution zooplankton. Although the relative closeness of the systems does not detect notable variations in the functional group structure (Pihl et al. 2002), their analysis denotes a certain functional redundancy level.

The fish assemblage composition found in coastal lagoons in the GT represents $32.1 \%$ of the continental taxa (548 species) and 13\% of all the near-shore fish fauna (1358 species) known for the TEP (Robertson \& Allen 2015). For Soconusco, Gómez-González et al. (2013) reported 67 species in CPE and 143 in CHP. Although the high value reported in the latter is due to a greater sampling effort with multiple fishing gears, its high richness implies some correspondence with the one reported here through standardized sampling with a cast net. It has been pointed out that the use of a single fishing gear results in a biased sample of a fish assemblage (Clement et al. 2014). However, in estuarine wetlands, cast nets are highly effective for achieving broad taxonomic representation in several shallow habitats and accurate estimates of abundances (Sheaves \& Johnston 2009). Other published lists correspond to Mar Muerto (66 species; Tapia-García et al. 1998), Oriental-Occidental (72 species; Chávez 1979) and Superior-Inferior lagoon systems (47 species; Tapia-García \& Mendoza-Rodríguez 2005).

Although the maximum similarity within each of the two lagoon complexes (Istmo and Soconusco) was $50 \%$, the classification and ordination analyses allowed to delineate relatively homogenous regional units, the two regional units would have a correspondence with the division of hydrological subsystems of the GT continental shelf proposed by Tapia-García et al. (2007). These authors claim that the northern subsystem (Oaxaqueño) is characterized by upwellings that influence dissolved oxygen, temperature, and nutrients. In contrast, in the southern (Chiapaneco) subsystem, a strong continental influence determines the biota pattern. The heterogeneity explained regionally here by the salinity, and mangrove area suggests relatively high beta diversity values between the two complexes and a certain homogeneity level within each one. The existence of dry and humid climatic zones and other systems' proximity seems to influence the similarity regionally. Although in terms of its mangrove fish fauna, the Tropical Eastern Pacific is considered as a single biogeographical unit (Castellanos-Galindo et al. 2013), the local heterogeneity of many areas can delineate ecoregions with distinguishable features.

Tropical and subtropical coastal lagoons present marked salinity fluctuations, defining a wet or dry phase (Velázquez-Velázquez et al. 2008). However, along climatic gradients (tropical/subtropical or arid/semi-arid), the ichthyofaunal composition may not show variations among localities, as has been observed in the northern coast of Brazil (Silva et al. 2017) or the Indo-Pacific (Kuo et al. 1999), in contrast, to warm/temperate transition zones where temperature can be a major factor in explaining zoogeographic 
patterns (Maree et al. 2000). In the GT, the low flow of freshwater that drain into the Istmo complex's lagoons leads to temporary hypersalinity conditions (Contreras et al. 1997). On the other hand, in the Soconusco complex, an increase in precipitation and the number of rivers can be key to defining lower salinity and a greater diversity of functional habitats for fish, which would also explain a greater number of residents and freshwater species.

For the GT, habitat heterogeneity may have a higher explanatory level than other simple variables considered key to associating richness patterns, such as area (Reséndez \& Kobelkowsky 1991). For the Neotropics' mangrove systems, the weight of the tidal dynamics and the biogeographic signature on the fish fauna's taxonomic and functional features have been demonstrated (Castellanos-Galindo \& Krumme 2015).

Chantuto-Panzacola is a relatively small system $\left(180 \mathrm{~km}^{2}\right)$ but with high fish species richness. Located in the most-humid zone of the GT, within the system drains more than six rivers that favor the development of an extensive mangrove area and freshwater swamps with emergent and submerged vegetation (Díaz-Ruiz et al. 2004). The mangrove, both locally (distance to the stand) and regional (area), was a highly explanatory variable for fish assemblages in the CHP. Species richness tends to increase with an increase in mangrove area as key resources such as food, shelter, and nursery habitats increase as a function of mangrove area (Sheaves \& Johnston 2009). Likewise, the mangrove species' type and dominance should be considered due to the differential use of mangrove microhabitats (prop roots or pneumatophores) by fishes (Rönnbäck et al. 1999).

Beta diversity can be a good indicator of a drastic hydrological fluctuation, which would favor the compositional change rate. Likewise, Atrill (2002) proposed that alpha diversity correlates negatively with salinity values. The effect of salinity on diversity has been observed at regional scales (Josefson 2009). Differences in alpha and beta values between the Istmo and Soconusco are consistent with this model; however, it is necessary to have time-scale assessments to understand the spatial diversity scene. Even assuming the possible bias derived from incomplete ichthyofaunal inventories, the gamma diversity in the coastal lagoons of the GT is comparatively higher (176) than the one estimated in Bahía de La Paz (67) in the lower Gulf of California [estimated value based on data from López-Rasgado (2012)], due to changes in the alpha and beta diversity between both regions. While in Bay of $\mathrm{La} \mathrm{Paz}$, there is a higher spatial turnover rate, in the GT, a higher average specific richness was observed, which could indicate the degree of heterogeneity within each system in this last region.
Beta diversity provides a measure of connectivity through a spatial or temporal gradient (Thrush et al. 2010). In aquatic environments, species' dispersal capacity favors the potential connectivity between local communities from a metacommunity. This concept has sought to unite local and regional patterns, their relative influence, and the ecological function of coexisting species (Leibold et al. 2004). Connectivity through dispersion will depend on the type of limits for each system. In many aquatic communities with discrete (ponds, lakes) or diffuse boundaries (reefs, rocky shores), this pattern has been relatively well explained (Caro et al. 2010, Drümmer et al. 2016). However, in systems that combine discrete and diffuse limits, such as intertidal environments, metacommunity features have rarely been modeled (Mouillot 2007, SanvicenteAñorve et al. 2011). Undoubtedly, alpha and beta's relative influence on gamma diversity should be a key element to be considered in local and regional management plans for coasts. The conservation of functional habitats and corridors that allow their connectivity could sustain coastal ecosystems' goods and services.

This work emphasizes the differences of speciesenvironment relationships in terms of scale and gradients of heterogeneity in lagoon systems associated with mangroves. In general, it was found that system variations can affect the species' relationship and their functionality. Although there is a general pattern in fish assemblage structure, local variations contribute to defining composition and distribution features at different scales. However, considering that mangroves can be affected by extraordinary environmental events (e.g. cyclones, El Niño-Southern Oscillation, changes in the tidal and rain regimes), some results presented here need to be taken with caution because the local variability between years could modify the fish assemblage. On the other hand, at the regional level, environmental and biotic databases from different studies can lead to biased interpretations of a system's ecological status. However, this approach to spatial patterns of diversity should be considered in developing coastal management plans. Likewise, it is deemed necessary to increase the studies that typify the mangrove-dependent ichthyofauna by differentiating it from other coastal soft-bottom assemblages to have solid elements to establish a more precise ecoregionalization.

\section{ACKNOWLEDGMENTS}

This work has been possible thanks to the logistic support of Museum of Zoology at Universidad de Ciencias y Artes de Chiapas and the grants from Fondo Mixto-Gobierno de Chiapas (FOMIX), Comisión 
Nacional para el Conocimiento y Uso de la Biodiversidad (CONABIO) and Consejo Nacional de Ciencia y Tecnología (CONACYT). Manuel Anzueto and Eduardo Urbina provided valuable help during fieldwork. Anonymous reviewers provided useful and important suggestions for improving the manuscript. Sampling of endangered species was performed under federal collection permits SGPA/DGVS/0429/08; SGPA/DGVS/00488/16; DGOPA.10863.221008.3028; DGOPA. 11679.10083071. CONACYT granted a scholarship in doctoral research for the first author. This work is dedicated to the memory of our beloved colleague and co-author Adán Enrique Gómez González ${ }^{\dagger}$.

\section{REFERENCES}

Anderson, M.J. 2001. A new method for non-parametric multivariate analysis of variance. Australian Journal of Ecology, 26: 32-46. doi: 10.1111/j.1442-9993.2001. 01070.pp.x

Anderson, M.J. 2006. Distance-based tests for homogeneity of multivariate dispersions. Biometrics, 62: 245253. doi: 10.1111/j.1541-0420.2005.00440.x

Anderson, M.J. \& Willis, T.J. 2003. Canonical analysis of principal coordinates: a useful method of constrained ordination for ecology. Ecology, 84: 511-525. doi: 10.1890/0012-9658(2003)084[0511:caopca]2.0.co;2

Anderson, M.J., Gorley, R.N. \& Clarke, K.R. 2008. PERMANOVA+ for PRIMER: guide to software and statistical methods. PRIMER-E, Plymouth.

Atrill, M.J. 2002. A testable linear model for diversity trends in estuaries. Journal of Animal Ecology, 71: 262-269. doi: 10.1046/j.1365-2656.2002.00593.x

Avilés-Torres, S., Schmitter-Soto, J.J. \& BarrientosMedina, R.C. 2001. Patrones espaciales de la riqueza de peces en lagunas costeras del sur de Quintana Roo, México. Hidrobiológica, 11: 141-148.

Bell, G., Lechowicz, M.J. \& Waterway, M.J. 2006. The comparative evidence relating to functional and neutral interpretations of biological communities. Ecology, 87: 1378-1386. doi: 10.1890/0012-9658 (2006)87[1378:TCERTF]2.0.CO;2

Bouchon-Navarro, Y., Bouchon, C., Louis, M. \& Legendre, P. 2005. Biogeographic patterns of coastal fish assemblages in the West Indies. Journal of Experimental Marine Biology and Ecology, 315: 3147. doi: 10.1016/j.jembe.2004.08.028

Caro, A.U., Navarrete, S.A. \& Castilla, J.C. 2010. Ecological convergence in a rocky intertidal shore metacommunity despite high spatial variability in recruitment regimes. Proceedings of the National Academy of Sciences, 107: 18528-18532. doi: 10.1073/pnas. 1007077107
Carranza-Edwards, A., Gutiérrez, M. \& Rodríguez, R. 1975. Unidades morfotectónicas continentales de las costas mexicanas. Anales del Instituto de Ciencias del Mar y Limnología, 2: 82-88.

Castellanos-Galindo, G.A. \& Krumme, U. 2015. Tides, salinity and biogeography affect fish assemblage structure and function in macrotidal mangroves in the Neotropics. Ecosystems, 18: 1165-1178. doi: 10.1007/ s10021-015-9887-4

Castellanos-Galindo, G.A., Krumme, U., Rubio, E.A. \& Saint-Paul, U. 2013. Spatial variability of mangrove fish assemblage composition in the Tropical Eastern Pacific Ocean. Reviews in Fish Biology and Fisheries, 23: 69-86. doi: 10.1007/s11160-012-9276-4

Castro-Aguirre, J.L. 1982. Los peces de las lagunas oriental y occidental, Oaxaca, México y sus relaciones con la temperatura y la salinidad. II. Análisis multifactorial. Anales de la Escuela Nacional de Ciencias Biológicas, 26: 85-100.

Castro-Aguirre, J.L., Espinosa-Pérez, H.S. \& SchmitterSoto, J.J. 1999. Ictiofauna estuarino-lagunar y vicaria de México. Limusa-Noriega/IPN, Mexico City.

Chase, J.M. \& Leibold, M.A. 2003. Ecological niches: interspecific interactions. The University of Chicago Press, Chicago.

Chávez, E. 1979. Análisis de la comunidad de una laguna costera en la costa sur occidental de México. Anales del Instituto de Ciencias del Mar y LimnologíaUNAM, 6: 15-44.

Chávez, E., Sevilla, A.M.L. \& Hidalgo, E. 1977. Estudio hidrográfico de dos lagunas costeras de Oaxaca. In: Manrique, F.A. (Ed.). Memorias del V Congreso Nacional de Oceanografía. ITESM/IPN, Guaymas, pp. 201-232.

Clement, T.A., Pangle, K., Uzarski, D.G. \& Murray, D.A. 2014. Effectiveness of fishing gears to assess fish assemblage size structure in small lake ecosystems. Fisheries Management and Ecology, 21: 211-219. doi: 10.1111/fme. 12069

Contreras, E.F., Castañeda, O.L. \& Torres, A.R. 1997. Hidrología, nutrientes y productividad primaria en las lagunas costeras del estado de Oaxaca, México. Hidrobiológica, 7: 9-17.

De la Lanza-Espino, G.J., Ortiz-Pérez, M.A. \& CarbajalPérez, J.L. 2013. Diferenciación hidrogeomorfológica de los ambientes costeros del Pacífico, del Golfo de México y del Mar Caribe. Investigaciones Geográficas, 81: 33-50. doi: 10.14350/rig.33375

Díaz-Ruiz, S., Aguirre-León, A. \& Cano-Quiroga, E. 2006. Evaluación ecológica de las comunidades de peces en dos sistemas lagunares estuarinos del sur de Chiapas, México. Hidrobiológica, 16: 197-210.

Díaz-Ruiz, S., Cano-Quiroga, E., Aguirre-León, A. \& Ortega-Bernal, R. 2004. Diversidad, abundancia y 
conjuntos ictiofaunisticos del sistema lagunar-estuarino Chantuto-Panzacola, Chiapas, México. Revista de Biología Tropical, 52: 187-199. doi: 10.15517/rbt. v52i1.14879

Drümmer, B., Ristau, K. \& Traunspurger, W. 2016. Varying patterns on varying scales: a metacommunity analysis of nematodes in European lakes. Plos One, 11: e0151866. doi: 10.1371/journal.pone.0151866

Eschmeyer, W.N., Fricke, R. \& Van der Laan, R. 2017. Catalog of fishes: genera, species, references. [http:// researcharchive.calacademy.org/research/Ichthyology /catalog/fishcatmai.asp.]. Reviewed: November 16, 2017.

Fischer, W., Krupp, F., Schneider, W., Sommer, C., Carpenter, K.E. \& Niem, V.H. 1995. Guía FAO para la identificación de especies para los fines de la pesca. FAO, Rome.

Froese, R. \& Pauly, D. (Eds.). 2016. FishBase. [www. fishbase.org]. Reviewed: October 16, 2016.

García, E. 2004. Modificaciones al sistema de clasificación climática de Köppen. Instituto de GeografíaUNAM, Mexico City.

Gómez-González, A.E., Velázquez-Velázquez, E., Rodiles-Hernández, R., González-Díaz, A.A., González-Acosta, A.F. \& Castro-Aguirre, J.L. 2012. Lista sistemática de la ictiofauna en la Reserva de la Biosfera La Encrucijada, Chiapas, México. Revista Mexicana de Biodiversidad, 83: 674-686. doi: 10.7550/ rmb.24468

Gotelli, N.J. \& Colwell, R.K. 2011. Estimating species richness. In: Magurran, A.E. \& McGill, B.J. (Eds.). Biological diversity. Frontiers in measurement and assessment. Oxford University Press, Oxford, pp. 39-54.

Halpern, B.S. 2003. The impact of marine reserves: do reserves work and does reserve size matter? Ecological Applications, 13: 117-137. doi: 10.1890/1051-0761 (2003)013[0117:TIOMRD]2.0.CO;2

Hammer, Ø., Harper, D.A.T. \& Ryan, P.D. 2001. PAST: paleontological statistics software package for education and data analyses. Palaeontologia Electronica, 4: 9.

Hubbell, S.P. 2001. The unified neutral theory of biodiversity and biogeography. Princeton University Press, Princeton.

Josefson, A.B. 2009. Additive partitioning of estuarine benthic macroinvertebrate diversity across multiple spatial scales. Marine Ecology Progress Series, 396: 283-292. doi: 10.3354/meps08375

Keddy, P.A. 1992. Assembly and response rules two goals for predictive community ecology. Journal of Vegetation Science, 3: 157-164. doi: 10.2307/3235676

Kuo, S.R., Lin, H.J. \& Shao, K.T. 1999. Fish assemblages in the mangrove creeks of northern and southern Taiwan. Estuaries, 22: 1004-1015. doi: 10.2307/ 1353079
Lacerda, L.D., Conde, J.E., Kjerfve, B., Alvarez-León, R., Alarcón, C. \& Polanía, J. 2002. American mangroves. In: Lacerda, D. (Ed.). Mangrove ecosystems: function and management. Springer, Heidelberg, pp. 1-62. doi: 10.1007/978-3-662-04713-2_1

Lankford, R.R. 1977. Coastal lagoons of Mexico. Their origin and classification. In: Wiley, M. (Ed.). Estuarine processes. Circulation, sediments, and transfer of material in the estuary. Academic Press, New York, pp. 182-215.

Legendre, P. \& Fortin, M.J. 1989. Spatial pattern and ecological analysis. Vegetatio, 80: 107-138. doi: 10.1007/BF00048036

Legendre, P. \& Legendre, L. 2012. Numerical ecology. Elsevier, Amsterdam.

Legendre, P., Mi, X., Ren, H., Ma, K., Yu, M., Sun, I.F. \& He, F. 2009. Partitioning beta diversity in a subtropical broad-level forest of China. Ecology, 90: 663-674. doi: 10.1890/07-1880.1

Leibold, M.A., Holyoak, M., Mouquet, N., Amarasekare, P., Chase J.M., Hoopes, M.F., et al. 2004. The metacommunity concept: a framework for multi-scale community ecology. Ecology Letters, 7: 601-613. doi: 10.1111/j.1461-0248.2004.00608.x

López-Rasgado, F.J. 2012. Estructura de la comunidad íctica de manglar en tres sistemas (Balandra, Enfermería y Zacatecas) y dos periodos (1980 y 2010) en relación con el grado de influencia antrópica. In: Pérez-Ramírez, M. \& Lluch-Cota, S.E. (Eds.). Biodiversidad y vulnerabilidad de ecosistemas costeros en Baja California Sur. Aportaciones de estudiantes de grado, posgrado y posdoctorado 2008-2012. CIBNOR, La Paz, pp. 375-398.

López-Vila, J.M., Schmitter-Soto, J.J., VelázquezVelázquez, E., Barba-Macías, E. \& Salgado-Ugarte, I.H. 2019. Young does not mean unstable: a trophic model for an estuarine lagoon system in the southern Mexican Pacific. Hydrobiologia, 827: 225-246. doi: 10.1007/s10750-018-3770-1

Magurran, A. 1988. Ecological diversity and its measurement. Princeton University Press, Princeton.

Magurran, A.E. \& Henderson, P.A. 2003. Explaining the excess of rare species in natural species abundance distributions. Nature, 422: 714-716. doi: 10.1038/ nature01547

Maree, R.C., Whitfield, A.K. \& Booth, A.J. 2000. Effect of water temperature on the biogeography of South African estuarine fishes associated with the subtropical/warm temperate subtraction zone. South African Journal of Science, 96:184-188.

Mendoza, E., Castillo-Rivera, M., Zárate-Hernández, R. \& Ortiz-Burgos, S. 2009. Seasonal variations in the diversity, abundance, and composition of species in an estuarine fish community in the Tropical Eastern 
Pacific, Mexico. Ichthyological Research, 56: 330339. doi: 10.1007/s10228-009-0102-5

Menezes, L.S., Müller, S.C. \& Overbeck, G.E. 2016. Scale-specific processes shape plant community patterns in subtropical coastal grasslands. Austral Ecology, 41: 65-73. doi: 10.1111/aec.12299

Miller, R.R., Minckley, W.L. \& Norris, S.M. 2009. Peces dulceacuícolas de México. Comisión Nacional para el Conocimiento y Uso de la Biodiversidad/Sociedad Ictiológica Mexicana A.C./El Colegio de la Frontera Sur/Desert Fishes Council, Mexico City.

Mouillot, D. 2007. Niche assembly vs. dispersal assembly rules in coastal fish metacommunities: implications for management of biodiversity in brackish lagoons. Journal of Applied Ecology, 44: 760-767. doi: 10.1111/ j.1365-2664.2007.01330.x

Myers, G.S. 1949. Salt-tolerance of fresh-water fish groups in relation to zoogeographical problems. Bijdragen tot de Dierkunde, 28: 315-322.

Pihl, L., Cattrijsse, A., Codling, I., Mathieson, S., McLusky, D.S. \& Roberts, C. 2002. Habitat use by fishes in estuaries and other brackish areas. In: Elliott, M. \& Hemingway, K. (Eds.). Fishes in estuaries. Blackwell Science, Oxford, pp. 10-53.

Polidoro, B.A., Carpenter, K.E., Dahdouh-Guebas, F., Ellison, J.C., Koedam, N.E. \& Yong, J.W.H. 2013. Global patterns of mangrove extinction risk: implications for ecosystem services and biodiversity loss. In: Maslo, B. \& Lockwood, J.L. (Eds.). Coastal conservation. Cambridge University Press, Cambridge, pp. 15-36.

Prasad, M.B.K., Dittmar, T. \& Ramanathan, A. 2010. Organic matter and mangrove productivity. In: Ramanathan, A., Bhattacharya, P., Dittmar, T., Prasad, M.B.K. \& Neupane, B.R. (Eds.). Management and sustainable development of coastal zone environments. Springer, Dordrecht, pp. 175-193.

Raz-Guzmán, A. \& Huidobro, L. 2002. Fish communities in two environmentally different estuarine systems of Mexico. Journal of Fish Biology, 61: 182-195. doi: 10.1111/j.1095.649.2002.tb01770.x

Reséndez, A. \& Kobelkowsky, A. 1991. Ictiofauna de los sistemas lagunares costeros del Golfo de México. Universidad y Ciencia. 8: 91-110.

Robertson, D.R. \& Allen, G.R. 2015. Shorefishes of the tropical eastern Pacific: online information system. Version 2.0. [http://www.biogeodb.stri.si.edu/sftep/ en/pages]. Reviewed: Octuber 16, 2016.

Romero-Berny, E.I., Velázquez-Velázquez, E., AnzuetoCalvo, M.J., Urbina-Trejo, E. \& Schmitter-Soto, J.J. 2018. The fish fauna of three lagoon-estuarine systems in the northeastern Gulf of Tehuantepec, Mexican south Pacific. Revista Mexicana de Biodiversidad, 89: 87-100. doi: 10.22201/ib.20078706e.2018.1.1927.
Rönnbäck, P., Troell, M., Kautsky, N. \& Primavera, J.H. 1999. Distribution pattern of shrimps and fish among Avicennia and Rhizophora microhabitats in the Pagbilao mangroves, Philippines. Estuarine, Coastal and Shelf Science, 48: 223-234. doi: 10.1006/ecss. 1998.0415

Sanvicente-Añorve, L., Sánchez-Ramírez, M., OcañaLuna, A., Flores-Coto, C. \& Ordoñez-López, U. 2011. Metacommunity structure of estuarine fish larvae: the role of local and regional processes. Journal of Plankton Research, 33: 179-194. doi: 10.1093/plankt/ fbq098

Sætersdal, G., Bianchi, G., Strømme, T. \& Venema, S.C. 1999. The Dr. Fridjoft Nansen Programme 1975-1993. Investigations of fishery resources in developing countries. FAO Fisheries Technical Papers No. 391: $493 \mathrm{pp}$.

Schluter, D. \& Ricklefs, R.E. 1993. Species diversity: an introduction to the problem. In: Ricklefs, R.E. \& Schluter, D. (Eds.). Species diversity in ecological communities: historical and geographical perspectives. University of Chicago Press, Chicago, pp. 1-10.

Sheaves, M. 2006. Scale-dependent variation in composition of fish fauna among tropical estuarine sandy embayments. Marine Ecology Progress Series, 310: 173-184. doi: 10.3354/meps310173

Sheaves, M. 2012. Ecosystem equivalence and the ability to generalize: insights from global consistencies in mangrove fish assemblages. Marine Ecology Progress Series, 461: 137-149. doi: 10.3354/meps09774

Sheaves, M. \& Johnston, R. 2009. Ecological drivers of spatial variability among fish fauna of 21 tropical Australian estuaries. Marine Ecology Progress Series, 385: 245-260. doi: 10.3354/meps 08040

Silva, J.M.G., Castro, A.C.L. \& Saint-Paul, U. 2017. Comparative analysis of distribution of intertidal fish assemblages in different estuarine systems on northern coast of Brazil. Journal of Sustainable Development, 10: 26-36. doi: 10.5539/jsd.v10n2p26

Sokal, R.R. \& Rohlf, F.J. 1995. Biometry: the principles and practice of statistics in biological research. W.H. Freeman, New York.

Tapia-García, M. \& Mendoza-Rodríguez, R. 2005. Composición y abundancia de la ictiofauna de las lagunas Superior e Inferior, Oaxaca, México. Actualidades Biológicas, 27: 57-65.

Tapia-García, M., Suárez-Núñez, C., Cerdenares-Ladrón de Guevara, C., Macuitl-Montes, M.C. \& GarcíaAbad, M.C. 1998. Composición y distribución de la ictiofauna en la Laguna del Mar Muerto, Pacífico Mexicano. Revista de Biología Tropical, 46: 277-284. 
Tapia-García, M., García-Abad, M.C., Carranza-Edwards, A. \& Vázquez-Gutiérrez, F. 2007. Environmental characterization of the continental shelf of the Gulf of Tehuantepec, Mexico. Geofísica Internacional, 46: 249-260.

Thrush, S.F., Hewitt, J.E., Cummings, V.J., Norkko, A. \& Chiantore, M. 2010. $\beta$-Diversity and species accumulation in Antarctic coastal benthos: influence of habitat, distance, and productivity on ecological connectivity. Plos One, 5: e11899. doi: 10.1371/ journal.pone.0011899

Torres-Orozco, B.R. 1994. Los peces. In: De la Lanza, E.G. \& Cáceres, C.M. (Eds.). Lagunas costeras y el litoral mexicano. UABCS, La Paz, pp. 270-304.

Tovilla-Hernández, C. \& Romero-Berny, E.I. 2012. Diagnóstico estructural de los manglares de Chiapas y Oaxaca. In: Sánchez, A.J., Chiappa-Carrara, X. \& Brito-Pérez, R. (Eds.). Recursos acuáticos costeros del sureste. RECORECOS/UNAM, Yucatán, pp. 257279.

Tovilla-Hernández, C., Ovalle-Estrada, F., De la PresaPérez, J.C., De la Cruz-Montes, G. \& De la CruzMontes, E. 2011. Inventario y monitoreo del estado actual de los bosques de manglar de Chiapas y Oaxaca. ECOSUR/CONABIO, Mexico City.

Tovilla-Hernández, C., Salas-Roblero, R.L., De la PresaPérez, J.C., Romero-Berny, E.I., Ovalle-Estrada, F., Gómez-Ortega, R., et al. 2007. Inventario forestal de los bosques de manglar de la costa de Chiapas. ECOSUR/COCYTECH, San Cristóbal de las Casas.

Vasconcelos, R.P., Henriques, S., França, S., Pasquaud, S., Cardoso, I., Laborde, M. \& Cabral, H.N. 2015. Global patterns and predictors of fish species richness in estuaries. Journal of Animal Ecology, 84: 13311341. doi: 10.1111/1365-2656.12372

Received: 4 December 2019; Accepted: 1 September 2020
Velázquez-Velázquez, E., Vega-Cendejas, M.E. \& Navarro-Alberto, J. 2008. Spatial and temporal variation of fish assemblages in a coastal lagoon of the Biosphere Reserve La Encrucijada, Chiapas, Mexico. Revista de Biología Tropical, 56: 557-574. doi: 10.15517/RBT.V56I2.5608

Whitfield, A.K. 1999. Ichthyofaunal assemblages in estuaries: a South African case of study. Reviews in Fish Biology and Fisheries, 9: 151-186. doi: 10.1023/ A: 1008994405375

Wilkinson, T., Wiken E., Bezaury-Creel J., Hourigan T., Agardy T., Herrman H., et al. 2009. Marine ecoregions of North America. Commission for Environmental Cooperation, Montreal.

Wolff, M. 2009. Tropical waters and their living resources: ecology, assessment and management. Hauschild Verlag, Bremen.

Yáñez-Arancibia, A., Lara-Domínguez, A.L., AguirreLeón, A., Díaz-Ruiz, S., Amezcua-Linares, F., Flores, D. \& Chavance, P. 1985. Ecology of dominant fish population in tropical estuaries: Environmental factors regulating biological strategies and production. In: Yáñez-Arancibia, A. (Ed.). Fish community ecology in estuaries and coastal lagoons: towards an ecosystem integration. Editorial Universitaria-UNAM, Mexico City, pp. 311-366.

Zapata, F.A. \& Robertson, D.R. 2007. How many species of shore fishes are there in the Tropical Eastern Pacific? Journal of Biogeography, 34: 38-51. doi: 10.1111/j.1365-2699.2006.01586.x 